\title{
Electrochemically-Gated Field-Effect Transistor with Indium Tin Oxide Nanoparticles as Active Layer
}

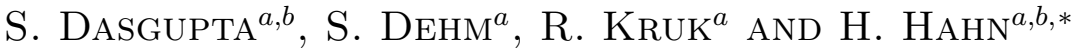 \\ ${ }^{a}$ Institute of Nanotechnology, Forschungszentrum Karlsruhe GmbH, P.O. Box 3640, D-76021 Karlsruhe, Germany \\ ${ }^{b}$ Center for Functional Nanostructures, Universität Karlsruhe, 76131 Karlsruhe, Germany
}

\begin{abstract}
An electrochemically-gated junction field-effect transistor with metallic conducting indium tin oxide nanoparticle array as active layer is reported. Fabrication of a field-effect device with a degenerative semiconductor like indium tin oxide (carrier concentration $10^{20}-10^{21} \mathrm{~cm}^{-3}$ ) is possible by exploiting the high surface-to-volume ratio of nanoparticles and high surface charge density achievable by electrochemical gating. The on/off ratio obtained is 325 although the applied potential was restricted to the capacitive double layer region (to ensure high repeatability) without allowing redox reactions to take place at the interface.
\end{abstract}

PACS numbers: 72.15.Eb, 73.63.Bd, 81.07.Bc, 82.45.Vp, 85.30.Tv

\section{Introduction}

Tailoring of material properties reversibly with an applied field (field-effect devices) at the surface is well known for semiconductors, conducting polymers [1-3] and nanotubes $[4,5]$. In contrast, tuning of physical properties to obtain measurable change as a function of applied field becomes extremely difficult for metals or high conducting oxides (degenerated semiconductors) due to their very large free-carrier concentration. In case of metals $\left(10^{22} \mathrm{~cm}^{-3}\right)$ or highly doped semiconductors $\left(10^{20}-10^{21} \mathrm{~cm}^{-3}\right)$ the high free carrier concentration limits the mean field penetration to only one or a few monolayers, respectively. Consequently, a high surface-to-volume ratio and a very high surface charge density is necessary to obtain a modulation in physical properties. Reversible tuning of the electron transport in thin gold films [6] and nanoporous [7] bulk gold has been reported recently. Although the observed variation in resistance was only a few percent, the understanding encouraged to look for different systems with higher effect-size and potential for device fabrication. The demonstration of tuning of electron transport of oxides with metallic conductivity in the present study opens new possibilities to alter physical properties of unconventional systems reversibly and dynamically. For instance, a field-effect transistor (FET) device with metallic conductivity was always a dream in order to have a high on-state current for a submicron-size device. In 2004, Rotkin and Hess [8] proposed the possibility of such a device using metallic carbon nanotubes (CNT) as channel material. While no experimental evidence of a metallic carbon nanotube

\footnotetext{
* corresponding author; e-mail: horst.hahn@int.fzk.de
}

FET has been demonstrated so far, in this paper, we report an alternative metallic conducting channel FET consisting of an oxide nanoparticle channel and an electrolyte as the gate dielectric.

In the present study, we have used indium tin oxide (ITO) nanoparticles as active layer and a non-aqueous electrolyte to provide the charges at the interface of the ITO nanoparticles. Similar to the work presented here using a liquid electrolyte, an all solid state device can also be fabricated using a solid polymer electrolyte as a gate dielectric [9]. The major advantage of the use of electrochemical gating is the orders of magnitude higher charge on the channel compared to any existing gate dielectrics and as a result the necessary gate voltage is reduced to less than one volt. Moreover, an electrolyte offers the additional advantage for a nanoparticle channel transistor as it easily penetrates the nanoporous network to reach all available surfaces of the nanoparticle array. Consequently, the applied electric field surrounds the surface of each individual nanoparticle and the necks between the nanoparticles as opposed to a field penetration just from one side in case of a dielectric gating.

\section{Experimental}

\subsection{Device fabrication}

The sample geometry is shown in Fig 1 . The contact leads are made of $310 \mathrm{~nm}$ thick ITO films (dark in Fig. 1) on a glass (bright in Fig. 1) substrate. The device fabrication starts with a $310 \mathrm{~nm}$ thick sputtered ITO film on high quality float glass. The leads are prepared using ebeam lithography on spin coated poly(methyl methacrylate) (PMMA) and ion etching. Subsequently, a second lithography step to create a $8 \times 2 \mu \mathrm{m}^{2}$ window for 


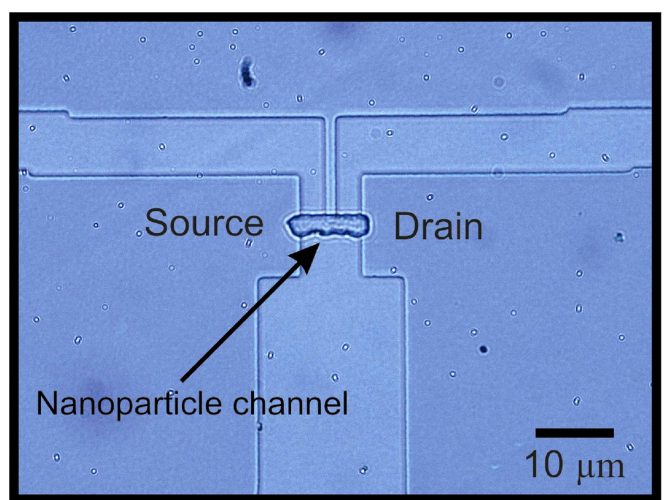

Fig. 1. Optical image of the device showing the source, drain made of sputtered ITO film, and the channel made of ITO nanoparticles.

spin coating of the ITO nanoparticles dispersion (Evonik $\mathrm{GmbH}$ ) follows. After the spin coating of the nanoparticle dispersion a low temperature $\left(185^{\circ} \mathrm{C}\right)$ annealing for $1 \mathrm{~h}$, prior to the PMMA layer lift-off with acetone, is performed. The whole structure is then annealed in air for $2 \mathrm{~h}$ at $500^{\circ} \mathrm{C}$ to eliminate the organic surfactants from the ITO surfaces. $0.1 \mathrm{M} \mathrm{NaClO}_{4}$ in propylene carbonate is used as the electrolyte. High surface area $\left(>1000 \mathrm{~m}^{2} / \mathrm{g}\right)$ activated carbon cloth (kynol) is used as a counter and a platinum wire $(99.99 \%$, Chempur) is used as a pseudoreference electrode.

\subsection{Methods}

The electrochemical measurements are done using an Autolab 302 potentiostat. For electrical measurements a Keithley 2182A nanovoltmeter, a Keithley 6221 DC and AC current source and a HP 3245A universal source are used.

\section{Results and discussion}

All the measurements were carried out at room temperature. The cyclovoltametry was performed with a glass substrate entirely spin coated with ITO nanoparticles to determine the potential window with negligible adsorption. The area of the nanoparticle channel in this device is many orders of magnitude lower than the contact leads. Therefore, the majority of the charging current for the entire device results from the leads and not from the ITO nanoparticles. Consequently, to determine the electrochemical stability and capacitive potential window of the nanoparticle a $25 \mathrm{~mm} \times 25 \mathrm{~mm}$ glass substrate entirely spin coated with nanoparticle dispersion was used.

Cyclovoltammograms were recorded with a very slow scan rate of $5 \times 10^{-4} \mathrm{~V} \mathrm{~s}^{-1}$ (Fig. 2a). The accumulated charge is determined by integrating the charging current. The identical potential window with the same scan rate is used during the measurement of the resistance change of the ITO nanoparticle device. A constant current of
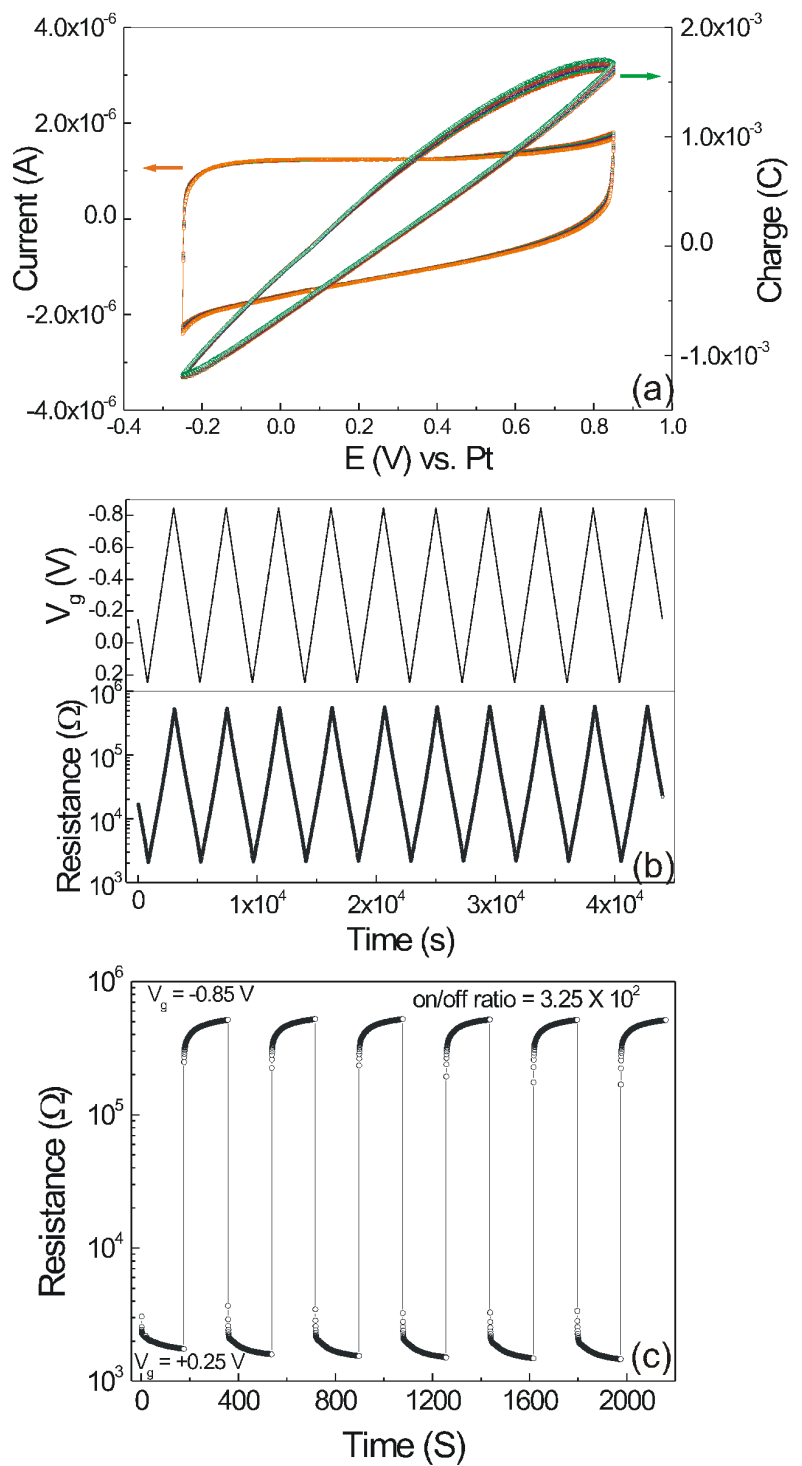

Fig. 2. (a) Cyclovoltammogram of dispersed ITO nanoparticles. (b) Change in resistance of the ITO channel when gate voltage is varied with a constant rate of $5 \times 10^{-4} \mathrm{~V} / \mathrm{s}$. (c) Change in the channel resistance in response to the potential pulses applied between the gate and channel.

$0.1 \mu \mathrm{A}$ is applied and the potential drop across two terminals is measured. More than two orders of magnitude change in resistance is observed when the potential of the working electrode is varied between -0.25 to $0.85 \mathrm{~V}$ $\left(V_{\mathrm{g}}=-0.85\right.$ to $\left.0.25 \mathrm{~V}\right)$ (Fig. $\left.2 \mathrm{~b}\right)$. At the negative potential of the working electrode (positive gate potential) positive ions (i.e. $\mathrm{Na}^{+}$ions) come close to the surface of the ITO nanoparticles. As a result, a redistribution of the electrons in the ITO nanoparticles occurs and electrons are attracted towards the surface to build the charge double layer. As a consequence, a negative electrode potential (positive gate potential) increases the carrier 
(electron) density of the ITO nanoparticles resulting in a decrease in the resistance of the channel. In case of a positive electrode potential (negative gate) negative ions (i.e. $\mathrm{ClO}_{4}^{-}$) form the charge double layer which repels the electrons away from the metal-electrolyte interface. Accordingly, the electron density of the channel decreases with a corresponding increase in the resistance. Therefore, the positive or negative ions of the charge double layer at the solid/electrolyte interface work as a gate and the channel gets narrower and broader with the negative and positive gate voltage, respectively, making the device analogous to a normally-on junction field effect transistor (JFET). Figure 2c shows the response of the device with the potential pulse of 0.25 and $-0.85 \mathrm{~V}$ applied after $180 \mathrm{~s}$. The resistance of the channel is measured with $25 \mathrm{~ms}$ time intervals. For the dry sample (before exposure to the electrolyte) the resistance of the channel is measured as $10 \pm 0.05 \mathrm{k} \Omega$. The resistivity of the nanoparticle assembly can be calculated readily as $0.025 \Omega \mathrm{cm}$. This value matches well with the value reported by Ederth et al. [10-12] for $16 \mathrm{~nm}$ ITO particles after annealing in air at $500^{\circ} \mathrm{C}$ for $2 \mathrm{~h}$.

Figure 3 shows the drain current-drain voltage $(I-V)$ characteristic of the device. The gate voltage is varied between 0.2 to $-0.8 \mathrm{~V}$ while the drain voltage is varied between $5 \mathrm{mV}$ to a maximum $980 \mathrm{mV}$. The separate measurement of the source and drain currents shows that the leakage current is two orders of magnitude lower than the drain/source current for all gate and drain voltage combinations. Another important device parameter which leads to low power operation is the subthreshold swing $(S)$. A small subthreshold swing is favored for switching transistors, with a theoretical limit of $60 \mathrm{mV}$ /decade. The $S$ value in the present device is only $415 \mathrm{mV} /$ decade. The small value of subthreshold swing of the present device is a result of the low gate voltage requirement of an electrochemically-gated device.

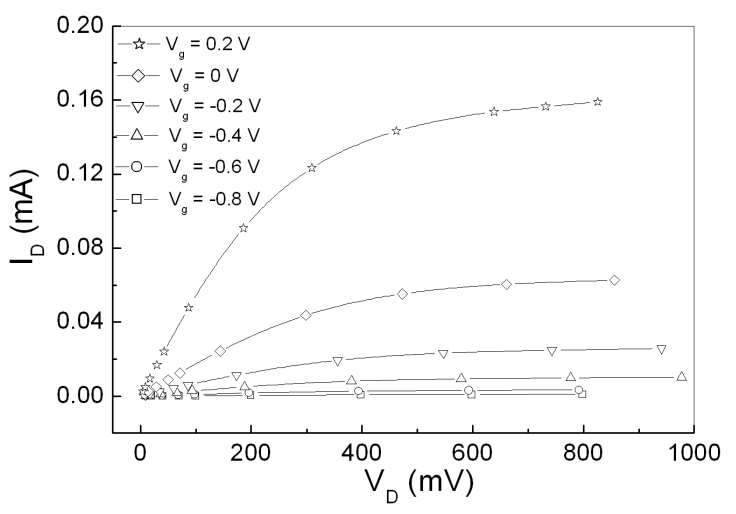

Fig. 3. The drain current-drain voltage characteristic of the device when the gate voltage varied between +200 and $-800 \mathrm{mV}$.

It is worthwhile to compare the difference between dielectric gating and electrochemical gating for the case of printable nanoparticulate structures. For a nanopar- ticle channel field-effect device with dielectric gating it is a prerequisite to have a quality interface between the dielectric and nanoparticles to ensure sufficient gating efficiency [13]. Therefore, a quality dispersion of nanoparticles with low agglomerate size is required. In contrast, the prototype FET device offered in the present study excludes the necessity to use quality dispersions as an electrolyte can penetrate the nanoporous network of a nanoparticle channel to form atomically smooth interfaces. With the possibility of using dispersions with higher agglomerate size and hence with a little or no surfactant ensures a reduction in annealing temperature to a much lower value to facilitate practical application. In spite of showing a very high mobility value [9] compared to organic semiconductors [14] or other nanoparticle channel transistors $[15,16]$ reported so far, the switching speed of the device is limited by the ionic mobility of the electrolyte. However, a faster device can be envisioned with an electrolyte of higher conductivity and a favorable geometry of the device (minimizing the channelgate distance).

Finally, it is necessary to compare a liquid and a polymeric solid electrolyte gating. Whereas a polymer based solid electrolyte produces an all-solid-state device, which is highly favorable for practical application, however, it is well known that even the best reported polymeric solid electrolyte possesses a conductivity orders of magnitude lower than the liquid counterpart. Therefore, it can be concluded that a liquid electrolyte gating would definitely be preferred for a field-effect device where the faster switching is essential.

\section{Summary}

In summary, an easy-to-fabricate, low voltage, electrochemical charge induced junction field-effect transistor (JFET) based on an ITO nanoparticle array (exhibiting metallic conductivity) is demonstrated. The observed on/off ratio is 325 . The present study suggests a new prospect of electronic devices from dispersions of near- metallic or degenerative semiconducting nanoparticles and an electrolyte as a gate dielectric. It is believed that a higher on/off ratio can easily be obtained with oxide nanoparticles with lower carrier density or by reducing the primary particle size. Furthermore, the control of the neck diameter between individual nanoparticles is believed to play a key role.

\section{Acknowledgments}

We acknowledge the help of Fornasier Heike and Dr. Dieter Mass of the Institute for Microsystem Technology for assistance in providing structured substrates. Financial support provided by the Deutsche Forschungsgemeinschaft (DFG) and the State of Baden-Württemberg through the DFG-Center for Functional Nanostructures (CFN) within sub-project D4.4. 


\section{References}

[1] J.D. Yuen, A.S. Dhoot, E.B. Namdas, N.E. Coates, M. Heeney, I. McCulloch, D. Moses, A.J. Heeger, J. Am. Chem. Soc. 129, 14367 (2007).

[2] M.J. Panzer, C.D. Frisbie, Adv. Funct. Mater. 16, 1051 (2006).

[3] M.J. Panzer, C.D. Frisbie, J. Am. Chem. Soc. 129 6599 (2007)

[4] G.P. Siddons, D. Merchin, J.H. Back, J.K. Jeong, M. Shim, Nano Lett. 4, 927 (2004).

[5] T. Ozel, A. Gaur, J.A. Rogers, M. Shim, Nano Lett. 5, 905 (2005)

[6] S. Dasgupta, R. Kruk, D. Ebke, A. Hütten, C. Bansal, H. Hahn, J. Appl. Phys. 104, 103707 (2008).

[7] A.K. Mishra, C. Bansal, H. Hahn, J. Appl. Phys. 103, 094308 (2008).

[8] S.V. Rotkin, K. Hess, Appl. Phys. Lett. 84, 3139 (2004).
[9] S. Dasgupta, S. Gottschalk, R. Kruk, H. Hahn, Nanotechnology 19, 435203 (2008).

[10] J. Ederth, P. Johnsson, G.A. Niklasson, A. Hoel, A. Hultåker, P. Heszler, C.G. Granqvist, A.R. van Doorn, M.J. Jongerius, D. Burgard, Phys. Rev. B 68, $155410(2003)$.

[11] J. Ederth, G.A. Niklasson, A. Hultåker, P. Heszler, C.G. Granqvist, A.R. van Doorn, M.J. Jongerius, D. Burgard, J. Appl. Phys. 93, 984 (2003).

[12] J. Ederth, A. Hultåker, G.A. Niklasson, P. Heszler, A.R. van Doorn, M.J. Jongerius, D. Burgard, C.G. Granqvist, Appl. Phys. A 81, 1363 (2005).

[13] K. Okamura, N. Mechau, D. Nikolova, H. Hahn, J. Appl. Phys. 93, 083105 (2008).

[14] H.E. Katz, Chem. Mater. 16, 4748 (2004).

[15] D.V. Talapin, C.B. Murray, Science 310, 86 (2005).

[16] T. Cui, Y. Liu, M. Zhu, Appl. Phys. Lett. 87, 183105 (2005). 